\title{
Atmospheric radiative heating due to aspherical coarse dust
}

\author{
AKINORI ITO ${ }^{1}$, ADEYEMI A. ADEBIYI ${ }^{2}$, YUE HUANG ${ }^{2}$ \\ AND JASPER F. KOK ${ }^{2}$ \\ ${ }^{1}$ JAMSTEC \\ ${ }^{2}$ University of California \\ Presenting Author: akinorii@jamstec.go.jp
}

Mineral dust aerosols cool and warm the atmosphere by scattering and absorbing both solar (short-wave: SW) and thermal (long-wave: LW) radiation. However, large uncertainties remain in dust radiative effects, largely due to differences in the dust size distribution and optical properties simulated in Earth system models. Recently, there has been increased attention paid to the importance of accurately predicting the abundance of coarse dust for the global energy balance.

Here, we improve the simulated dust properties with datasets that leverage measurements of size-resolved dust concentration and asphericity factor (improved simulation) in a coupled global chemical transport model (IMPACT) with a radiative transfer module (RRTMG) (default simulation). The global and annual average of dust aerosol optical depth at $550 \mathrm{~nm}\left(\mathrm{DAOD}_{550}\right)$ from the improved simulation falls within the range of a semiobservation-based estimate, in contrast to that of the default simulation. Improved agreement against semi-observation-based estimate of the radiative effect efficiency was obtained using less absorptive SW and more absorptive LW dust refractive indices. Our sensitivity simulations reveal that the improved simulation leads to a similar net global dust radiative effect at the Top Of Atmosphere (TOA) on a global scale to the default simulation but results in less cooling at the surface, because of enhanced LW warming by aspherical coarser dust. Our results thus suggest less atmospheric radiative heating due to aspherical dust with coarser size over the major source regions. 